\title{
ON THE SELFADJOINTNESS OF DIRAC OPERATORS WITH ANOMALOUS MAGNETIC MOMENT
}

\author{
F. GESZTESY, B. SIMON ${ }^{1}$ AND B. THALLER
}

\begin{abstract}
We provide a new proof of Behncke's remarkable result that the Coulombic Dirac equation with nonzero anomalous magnetic moment is essentially selfadjoint (on $C_{00}^{\infty}\left(R^{3}\right)^{4}$ ) for any value of the Coulomb charge.
\end{abstract}

In this note we shall consider Dirac operators. In the simplest version, these have the form

$$
H=\vec{\alpha} \cdot \vec{p}+m \beta+V
$$

where $\vec{p}=-i \vec{\nabla}$ on $L^{2}\left(R^{3}\right)$ and $H$ acts on $L^{2}\left(R^{3}, d^{3} x ; C^{4}\right) . \alpha, \beta$ are $4 \times 4$ matrices, written in terms of the conventional $2 \times 2$ Pauli sigma matrices, $\vec{\sigma}$, as $2 \times 2$ blocks of $2 \times 2$ matrices:

$$
\beta=\left(\begin{array}{c|c}
\mathbf{1} & 0 \\
\hline 0 & -\mathbf{1}
\end{array}\right), \quad \vec{\alpha}=\left(\begin{array}{c|c}
0 & \vec{\sigma} \\
\hline \overrightarrow{\boldsymbol{\sigma}} & 0
\end{array}\right) .
$$

It is well known (see e.g. $[1,8,9])$ that for $V=e|\vec{x}|^{-1},(1)$ is essentially selfadjoint on $C_{0}^{\infty}\left(R^{3}\right)^{4}$ if and only if $e \leqslant \sqrt{3 / 4}$, and strange spectral properties occur if $e>1$ [11]. Indeed, it has been speculated that these difficulties have physical significance for the stability of the world if superheavy nucleii with charge $Z>137$ exist (written back in conventional units $Z=e \alpha^{-1}$ with $\alpha$ the fine structure constant); see $[6,10]$ and the references therein. We feel that these speculations are ill founded for a number of reasons including the theme of this note.

Equation (1) corresponds to the equation for an electron with magnetic moment 1 (in units of Bohr magnetons), but it is known that the actual value is $1+\mu$ where $\mu=0.001159$ is called the anomalous magnetic moment [5] (understood from the point of view of quantum electrodynamics). Equation (1) in the presence of such an anomalous moment should be replaced by [13]

$$
H=\vec{\alpha} \cdot \vec{p}+m \beta+V-\frac{\mu}{2 m} \vec{\tau} \cdot \vec{\nabla} V
$$

with

$$
\vec{\tau}=\left(\begin{array}{c|c}
0 & i \vec{\sigma} \\
\hline-i \vec{\sigma} & 0
\end{array}\right)
$$

Received by the editors June 12, 1984.

1980 Mathematics Subject Classification. Primary 81C10, 35F10; Secondary 34B20, 47F05.

${ }^{1}$ Research partially supported by USNSF Grant MCS-81-20833. 
Recently, Behncke [3] (see also his papers [4, 16] and the earlier work of Barut and Kraus [2]), discovered the remarkable result that if $\mu \neq 0$ and $V=e|\vec{x}|^{-1}$, then (2) is essentially selfadjoint on $C_{00}^{\infty}\left(R^{3}\right)^{4}$ (here $C_{00}^{\infty}=C_{0}^{\infty}\left(R^{3} \backslash\{0\}\right.$ ) for any $e \neq 0$ ). Behncke's analysis depends essentially on the central symmetry of $V(\vec{x})=e|\vec{x}|^{-1}$ which allows one to write (2) as a direct sum of (two-component) ODE's. He analyzes these new operators by the well-developed selfadjointness techniques of such ODE's (see e.g. Weidman [15]). Our goal here is to prove Behncke's result using operator theoretic methods.

Absorbing $\mu / 2 m$ into $V$, (2) finally becomes

$$
H=\vec{\alpha} \cdot \vec{p}+m \beta+\frac{2 m}{\mu} V-\vec{\tau} \cdot \vec{\nabla} V .
$$

In order to study $\left(2^{\prime}\right)$, it turns out to be useful to introduce operators of the type

$$
C=\left(\begin{array}{cc}
0 & A_{-} \\
A_{+} & 0
\end{array}\right), \quad S=\left(\begin{array}{cc}
S_{+} & 0 \\
0 & S_{-}
\end{array}\right) .
$$

We first state (see Kato [7] for discussions of $A$-boundedness)

Proposition 1. $S$ is $C$-bounded with relative bound zero if and only if $S_{+}$is $A_{+}$-bounded and $S_{-}$is $A_{-}$-bounded, each with relative bound zero.

Proof. $\left\|C\left(\varphi_{+}, \varphi_{-}\right)\right\|^{2}=\left\|A_{+} \varphi_{+}\right\|^{2}+\left\|A_{-} \varphi_{-}\right\|^{2}$ and $\left\|S\left(\varphi_{+}, \varphi_{-}\right)\right\|^{2}=\left\|S_{+} \varphi_{+}\right\|^{2}+$ $\left\|S_{-} \varphi_{-}\right\|^{2}$.

This leaves the question of essential selfadjointness of operators of the form $C$.

Proposition 2. An operator of the form $C$ is essentially selfadjoint if and only if

(i) $\left(A_{-}\right)^{*}=\bar{A}_{+}$,

(ii) $\left(A_{+}\right)^{*}=\bar{A}_{-}$.

Moreover, (i) is equivalent to (ii).

Proof. The equivalence of (i) and (ii) follows from $X^{* *}=\bar{X}$ and $\overline{X^{*}}=X^{*}$. The first assertion follows from the observation that $\bar{C}=\left(\begin{array}{cc}0_{\bar{A}} & \bar{A}_{+} \\ 0\end{array}\right)$ and secondly from the easy calculation that $C^{*}=\left(\begin{array}{cc}0 & A^{*} * \\ A^{*} & 0\end{array}\right)$.

REMARK. Nelson has noticed [12] that the fact that $C=\left({ }_{A^{*}}^{0}{ }_{0}^{A}\right)$ is selfadjoint if $A$ is closed provides a trivial proof of von Neumann's theorem that $A^{*} A$ is densely defined as selfadjoint: Just notice that by the spectral theorem, if $C$ is self-adjoint, then $C^{2}$ is densely defined and selfadjoint.

The final abstract result that we require is:

Proposition 3. Let $A, B$ be operators so that $B \subset A^{*}, A[D(A)] \subset D(\bar{B})$ and $B A$ is essentially selfadjoint on $D(A)$. Then $\bar{B}=A^{*}$.

Proof. By the last proposition, it is sufficient to prove $C=\left(\begin{array}{ll}0 & A \\ B & 0\end{array}\right)$ is essentially selfadjoint on $D(B) \oplus D(A)$. Now $C^{*}=\left(\begin{array}{c}0 \\ A^{*}\end{array} 0^{B^{*}}\right)$ on $D\left(A^{*}\right) \oplus D\left(B^{*}\right)$. Suppose that $(u, v) \in D\left(A^{*}\right) \oplus D\left(B^{*}\right)$ with $A^{*} u= \pm i v, B^{*} v= \pm i u$. Let $\varphi \in D(A)$. Then

$$
(\bar{B} A \varphi, v)=\left(A \varphi, B^{*} v\right)= \pm i(A \varphi, u)= \pm i\left(\varphi, A^{*} u\right)=-(\varphi, v)
$$


so $v \perp(\bar{B} A+1)[D(A)]$, violating the assumed essential selfadjointness of the positive operator $\bar{B} A$. Thus $v$ and so $u$ equal zero. It follows that $C$ has zero deficiency indices so it is essentially selfadjoint.

The standard angular momentum decomposition of Dirac operators when $V(x)=$ $V(|x|)$, shows that the operator of $\left(2^{\prime}\right)$ is unitarily equivalent (under a transformation taking $C_{00}^{\infty}\left(R^{3}\right)$ to $\left.C_{0}^{\infty}(0, \infty)\right)$ to a direct sum of operators $H_{j, \sigma}$ indexed by $j=\frac{1}{2}$, $\frac{3}{2}, \ldots$ and a sign $\sigma= \pm$ (and with the space indexed by $j$ occurring $2 j+1$ times) with $H_{j, \sigma}$ acting on $L^{2}\left((0, \infty), d r ; C^{2}\right)$ by $[2,3]$

$$
H_{j, \boldsymbol{\sigma}}=\left(\begin{array}{cc}
S_{+, j, \sigma} & A_{-, j, \sigma} \\
A_{+, j, \sigma} & S_{-, j, \sigma}
\end{array}\right),
$$

where $S_{ \pm}=2 m V(r) / \mu \pm m$ and

$$
A_{ \pm}= \pm \frac{d}{d r}-\frac{\kappa(j, \sigma)}{r}-\frac{d V}{d r}
$$

where $\kappa(j, \sigma)=\sigma(j+1 / 2)$. Define

$$
W(r)=-\frac{\kappa(j, \sigma)}{r}-\frac{d V}{d r} .
$$

Then, on $C_{0}^{\infty}(0, \infty), A_{+}^{*} \supset A_{-}$and

$$
B_{ \pm}=\bar{A}_{ \pm} A_{\mp}=\frac{-d^{2}}{d r^{2}}+W^{2} \pm \frac{d W}{d r} \equiv \frac{-d^{2}}{d r^{2}}+V_{\text {eff }}^{ \pm}
$$

where $V_{\text {eff }}^{ \pm}=\left(V^{\prime}\right)^{2}+2 \kappa r^{-1} V^{\prime} \mp V^{\prime \prime}-\left(\kappa^{2} \pm \kappa\right) r^{-2}$. If $V=e|\vec{x}|^{-1}$, then $W \sim e|\vec{x}|^{-2}$ for small $|\vec{x}|$ and

$$
V_{\text {eff }}^{ \pm} \sim e^{2}|\vec{x}|^{-4}
$$

for small $|\vec{x}|$; so $(6)$ is essentially selfadjoint on $C_{0}^{\infty}(0, \infty)$ by well-known results (the usual argument is one-dimensional-see Reed-Simon [14, Theorem X.10], but there are multidimensional arguments which apply also-see Reed-Simon [14, Theorem X.30]). Moreover, by (7)

$$
V^{2} \leqslant \varepsilon V_{\text {eff }}^{ \pm}+C(\varepsilon)
$$

so $V^{2}$ is a form bounded perturbation of $B_{ \pm}$. Thus, by Proposition 1.3, we have proven Behncke's theorem:

THEOREM 5. If $\mu \neq 0$ and $V=e|\vec{x}|^{-1}(e \neq 0)$, then (2) is essentially selfadjoint on $C_{00}^{\infty}\left(R^{3}\right)^{4}$.

The difficulties with noncentral potentials is shown by the fact that while (7), (8) hold for each value of $j$, they are not uniform in $j$.

We end by noting that one can compare our proof with that of Behncke [3] by noting the conditions under which our respective arguments apply. Behncke requires:

(B1) $V, V^{\prime} \in L_{\text {loc }}^{2}(0, \infty), \mu \neq 0$;

(B2) $\operatorname{Sgn} V^{\prime}$ constant near 0 ; 
(B3) $\lim _{r \rightarrow 0^{+}}\left(r V^{\prime}(r)\right)=\infty=\lim _{r \rightarrow 0^{+}}\left|V^{\prime}(r) / V(r)\right|$.

Our proof requires (if one uses Wüst's Theorem [14, Theorem X.14] in borderline cases to add on $S$ )

(GST1) $V^{\prime \prime} \in L_{\text {loc }}^{2}(0, \infty), \mu \neq 0$,

(GST2) $V_{\text {eff }}^{+}(r) \geqslant 3 / 4 r^{2}-d$ for some $d$ and $r$ small or the same result for $V_{\text {eff }}^{-}$,

(GST3) $V_{\mathrm{eff}}^{ \pm}(r)-V^{2}(r) \geqslant-\left(4 r^{-2}\right)-d$ for some $d$ where $V_{\mathrm{eff}}^{ \pm}$are defined by (5), (6), i.e.,

$$
V_{\text {eff }}^{ \pm}=\left(V^{\prime}\right)^{2}+2 \kappa r^{-1} V^{\prime} \mp V^{\prime \prime}+\left(\kappa^{2} \pm \kappa\right) r^{-2} .
$$

$V^{\prime \prime} \in L_{\text {loc }}^{2}$ in (GST1) can be replaced by $V^{\prime \prime} \in L_{\text {loc }}^{1}(0, \infty)$ using standard techniques.

Since Behncke has no conditions on $V^{\prime \prime}$, his results are, in a sense, stronger. But curiously, he does not allow $V=0$ or $V=\left[\ln \left(|r|^{-1}+2\right)\right]^{\alpha}$ with $\alpha<1$ while we do.

One of us (F. Gesztesy) would like to thank R. Vogt, W. Luxemburg and B. Simon for the hospitality of Caltech during a visit in April 1983.

\section{REFERENCES}

1. M. Arai, On essential selfadjointness, distinguished selfadjoint extension and essential spectrum of Dirac operators with matrix valued potentials, Publ. Res. Inst. Math. Sci. 19 (1983), 33-57.

2. A. O. Barut and J. Kraus, Solution of the Dirac equation with Coulomb and magnetic moment interactions, J. Math. Phys. 17 (1976), 506-508.

3. H. Behncke, The Dirac equation with an anomalous magnetic moment, Math. Z. 174 (1980), 213-225.

4. Spectral properties of the Dirac equation with anomalous magnetic moment, preprint.

5. H. Frauenfelder and E. M. Henley, Subatomic physics, Prentice-Hall, Englewood Cliffs, N. J., 1974.

6. W. Greiner (ed.), Quantum electrodynamics of strong fields, Vol. B80, Nato Advanced Study Institute Series, Plenum, 1983.

7. T. Kato, Perturbation theory for linear operators, 2nd ed., Springer-Verlag, Berlin and New York, 1980.

8. M. Klaus and R. Wüst, Characterization and uniqueness of distinguished selfadjoint extensions of Dirac operators, Comm. Math. Phys. 64 (1979), 171-176.

9. J. J. Landgren and P. A. Rejto, On a theorem of Jörgens and Chernoff concerning essential self-adjointness of Dirac operators, J. Reine Angew. Math. 332 (1981), 1-14.

10. B. Müller and W. Greiner, The physics of strong fields in quantum electrodynamics and general relativity, Acta Phys. Austriaca Suppl. 18 (1977), 153-384.

11. H. Narnhofer, Acta Phys. Austriaca 40 (1974), 306-322.

12. E. Nelson, unpublished.

13. W. Pauli, Die allgemeinen Prinzipien der Wellenmechanik, Encyclopedia of Physics V/1 (S. Flügge, ed.), Springer, Berlin and New York, 1958.

14. M. Reed and B. Simon, Methods of modern mathematical physics. II. Fourier analysis, self-adjointness, Academic Press, New York, 1975.

15. J. Weidmann, Oszillationsmethoden für Systeme gewöhnlicher Differentialgleichungen, Math. Z. 119 (1971), 349-373.

16. H. Behncke, Dirac equation with an anomalous magnetic moment. II, Ordinary and Partial Differential Equations (W. N. Everitt and B. D. Sleeman, eds.), Lecture Notes in Math., Vol. 964, Springer, Berlin and New York, 1982, pp. 77-85.

Institute for Theoretical Physics, University of Leuven, B - 3030 Leuven, Belgium

Division of Physics, Mathematics and Astronomy, Caltech, Pasadena, California 91125

Institute of Mathematics I, Free University of Berlin, D - 1000 Berlin 33, Federal Republic OF GERMANY 